- Original Article

\title{
A Longitudinal Study on the Effects of Negative Rearing Experiences on Adolescents' Social Withdrawal and Aggression
}

\author{
Kyung-Suk Lee', Ok-Joo Choi' ${ }^{2}$ Joon-Ho Kim ${ }^{3, *}$ \\ 'Department of Counseling Psychology, Seoul University of Buddhism, Seoul, Korea \\ ${ }^{2}$ Department of Social Welfare, Konkuk University, Seoul, Korea \\ ${ }^{3}$ Department of Business Administration, Sejong University, Seoul, Korea
}

Background: Children who have experienced negative rearing behaviors show a lack of self-confidence due to emotional instability and are reserved in interpersonal relationships. This can lead to failure in social adaptation and a high risk of depression, suicide, criminal acts, and anti-social behaviors. Therefore, this study aims to analyze the effects of experiencing negative parental rearing behaviors, such as neglect and abuse, on adolescents' social withdrawal and aggression, by utilizing multivariate latent growth models.

Methods: Data from the Korean Children and Youth Panel Study (KCYPS), a survey conducted by the National Youth Policy Institute targeting a cohort of three different age groups (grade 1, grade 4, and grade 7), from 2010 to 2016 was used. Multi-stage stratified sampling methods were used in the KCYPS, which surveyed the students and parents of the selected grade levels. This study analyzed the data for grade 7 , from second year (grade 8) to fourth year (grade 10).

Results: Negative rearing experiences had a significant effect on social withdrawal and aggression, and this influence was shown to persist over the long term.

Conclusion: This study examined the long-term developmental trajectory in the relationship between risk factors for adolescent development. Furthermore, the relationship between risk factors was shown to have not only short term but long-term effects as well, which reinforces the limitations of previous studies.

Keywords: Negative Rearing Experiences; Social Withdrawal; Aggression; Multivariate Latent Growth Models 


\section{INTRODUCTION}

During adolescence, integration of psychological autonomy and selfidentity occurs internally; externally, it involves developmental tasks such as entering secondary school and producing academic achievements. ${ }^{1)}$ A significant number of physical, psychological, and social changes are experienced that are accompanied by many internal and external conflicts. ${ }^{2)}$ Such internal and external changes can cause psychosocial problems, and they can be important indicators for predicting various behavioral problems of adulthood. ${ }^{3)}$

Among adolescent psychosocial problems, externalizing problems are distinguished by aggression due to under-control, including behavioral problems such as delinquency, while internalizing problems due to over-control result in depression, anxiety, and social withdrawal. ${ }^{4}{ }^{4}$ Internalizing problems have particularly been discussed as causing problems in cognitive, interpersonal, and learning areas. However, the majority of previous studies on adolescents have focused on externalizing problems, ${ }^{5)}$ which suggests the need for more studies on internalizing problems.

There have been studies on internalizing problems regarding depression and anxiety. ${ }^{6,7)}$ However, social withdrawal is another representative internalizing problem, which has been insufficiently studied. Social withdrawal indicates all types of behaviors and attitudes in which one seeks to be alone when facing social situations, and also indicates being shy or suppressed, having low energy, and avoiding social interactions. ${ }^{4)}$ Such social withdrawal is strongly related to low selfesteem, negative self-concept, and high depression and anxiety, creating vulnerability toward interpersonal relationships, behavioral problems, and peer rejection. These problems of adolescents can lead to social isolation, avoidant personality disorder, academic failure, unemployment, suicide attempts, and criminal behaviors in adulthood. ${ }^{8)}$ This is because internalizing problems of social withdrawal have a high probability of developing into externalizing problems of aggression and delinquency, over the long term. ${ }^{9)}$

The rearing behavior of parents is a crucial factor explaining the child's social withdrawal, which critically effects the adolescent's development of personality and social skills. This is also an important factor for social adaptation in adulthood. ${ }^{10)}$ Especially, negative parental rearing experience can cause social withdrawal of the child. ${ }^{11)}$ Children with negative rearing experiences may experience negative emotions such as frustration, fear, low self-control, and impulsivity that can lead to social withdrawal. ${ }^{11)}$

Parents' rearing behavior and social withdrawal are important predictive factors explaining adolescent aggression. According to attachment theory, having stable or unstable attachments with parents during infancy can have long-term effects. ${ }^{12)}$ Stable attachment positively influences social skill development. However, the opposite kind of attachment can cause external problems such as aggression, ultimately leading to problematic behaviors. ${ }^{13)}$

In conclusion, children who have experienced negative rearing behaviors show a lack of self-confidence due to emotional instability and are reserved in interpersonal relationships. This can lead to failure in social adaptation and high risks of depression, suicide, criminal acts, and anti-social behaviors. ${ }^{10,14-18)}$ Particularly, Korean adolescents experience excessive academic stress and are often deprived of social interactions with their family or peers, in terms of quality and quantity. This creates a very high probability of causing problems of social withdrawal. $^{19)}$

However, there are still insufficient studies that longitudinally analyze problems of social withdrawal among adolescents that may change dynamically over the course of adolescent development. Furthermore, in the few studies that have longitudinally analyzed social withdrawal, the developmental trajectory of social withdrawal was found to have an effect on propensity for avoiding risks, unstable relationships with peers, and depression., ${ }^{82022)}$ Therefore, there is an urgent need for systematic studies on how adolescents' social withdrawal problems change over time and the variables that affect this trajectory.

In this study, firstly, the developmental path of adolescents' social withdrawal over a period of 3 years was determined. Secondly, parental rearing behavior was considered as a factor that could affect the developmental trajectory of social withdrawal, and the relationship between the two was explored. Thirdly, the study aimed to analyze the effects of the developmental trajectory of social withdrawal on externalizing problems of aggression. This study is significant in the fact that emotional and behavioral aspects of dynamically changing adolescents were explored in depth.

\section{METHODS}

\section{Study Target}

In this study, the developmental trajectory of adolescents' negative rearing experiences, social withdrawal, and aggression were observed. Furthermore, the effects of negative rearing experiences on social withdrawal and aggression, and the effects of social withdrawal on aggression were analyzed. The factors influencing adolescent development were considered from a long-term perspective, in order to provide practical information about the normal development of adolescents.

Therefore, in this study, data from the Korean Children and Youth Panel Study (KCYPS) was used, a survey conducted by the National Youth Policy Institute targeting a cohort of three different age groups (grade 1, grade 4, and grade 7), from 2010 to 2016. Multi-stage stratified sampling methods were used in the KCYPS, which surveyed the students and parents of the selected grade levels. The present study analyzed data for grade 7 , from second year (grade 8) to fourth year (grade 10). A total of 2,351 cases were observed, and cases without responses were excluded, leaving the following number of cases in the analysis: 2,280 students for the second year; 2,259 students for the third year; and 2,108 students for the fourth year. The gender of study participants were as follows. Males were 1,152 (50.5\%) for the second year, 1,140 (50.5\%) for the third year, and 1,075 (51.0\%) for the fourth year. Fe- 
males were 1,128 (49.5\%) for the second year, 1,119 (49.5\%) for the third year, and 1,033 (49.0\%) for the fourth year.

\section{Measures}

\section{1) Negative rearing}

For negative rearing, measures of abuse and neglect were summed and an average was taken. The measure of abuse and neglect was constructed by referring to studies on child abuse by $\mathrm{Huh}^{23)}$ and Kim. ${ }^{24)}$ Four questions were used, including "I get scorned excessively when I do something wrong," with a 4-point scale as follows: 1=always, $2=$ sometimes, $3=$ rarely, and $4=$ never. For ease of interpretation, the scores were reversed, so that higher scores indicated higher levels of abuse. For the neglect measure, four questions including "My parents consider me to be more important than their work" were scored using a 4-point scale ( $1=$ =always, $2=$ sometimes, $3=$ rarely, and $4=$ never). Cronbach's $\alpha$ values for the questions regarding negative rearing experiences were as follows: 0.736 for second year, 0.753 for third year, and 0.785 for fourth year.

\section{2) Social withdrawal}

The study by Kim and $\mathrm{Kim}^{25)}$ was referred to for the measure of social withdrawal, that was constructed after removing repetitive questions and modifying the questions. Five questions were constructed including "I feel uncomfortable when there are a lot of people around me," and a 4-point scale (1=always, $2=$ =sometimes, $3=$ rarely, and 4=never) was used. For ease of interpretation, scores were reversed, so that higher scores would indicate higher levels of social withdrawal. Cron- bach's $\alpha$ values for the questions regarding social withdrawal were as follows: 0.850 for second year, 0.883 for third year, and 0.880 for fourth year.

\section{3) Aggression}

For the measure of aggression, the KCYPS data was used, which modified the measure developed by Cho and Lim. ${ }^{26)}$ For the measure of aggression, 6 questions were used, including "I get into fights over small things," with a 4-point scale (1=always, $2=$ =sometimes, $3=$ rarely, and $4=$ never). For ease of interpretation, scores were reversed, so that higher scores would indicate higher levels of aggression. Cronbach's $\alpha$ values for the questions regarding aggression were as follows: 0.810 for second year, 0.811 for third year, and 0.785 for fourth year.

\section{Research Model and Analysis Method}

In this study, a research model was constructed as shown in Figure 1, based on theoretical observations and a review of previous studies. The research model shows the developmental trajectory of adolescents' negative rearing experiences, social withdrawal, and aggression. As discussed in previous studies, a causal relationship was observed between the developmental trajectory of adolescents' negative rearing experiences, social withdrawal, and aggression.

The analysis process of this study consisted of the following steps. First, in order to observe the changing patterns of major variables in this study, the levels of the variables and the relationships between them at each time point were analyzed. For this, means and standard deviations for negative rearing, social withdrawal, and aggression were calculated for each time point, and correlational analysis was per-

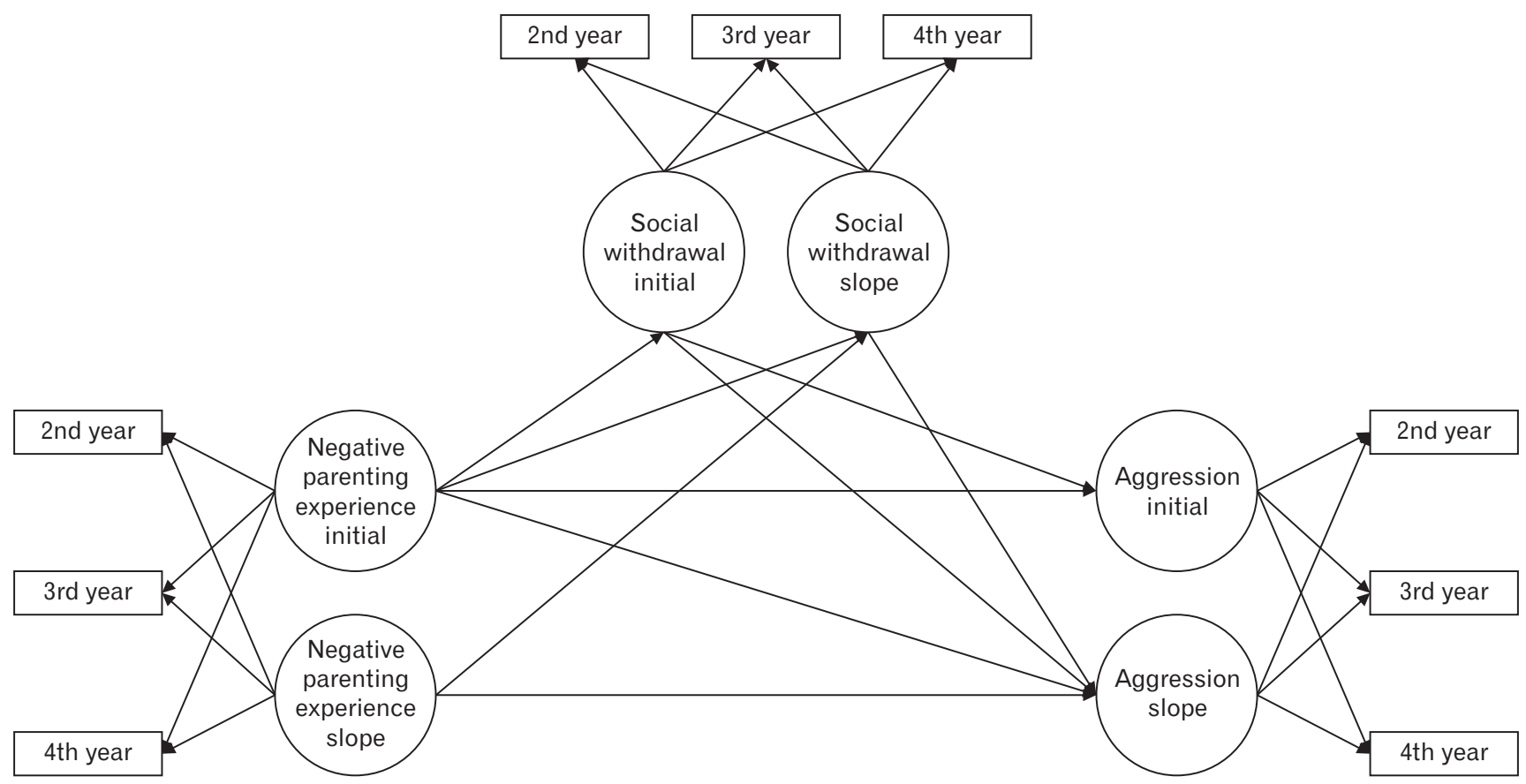

Figure 1. Research model. 
formed between each variable. Second, for the estimation of change functions due to time elapsed, latent growth modeling was conducted. Since this study consists of three time points, a no-change model and a linear model were set as competing models. A goodness of fit index was used to provide objective criteria for the simplicity and explanatory power of the model. Third, the variables in the change model were examined, and a latent model analysis for each variable was obtained to investigate the causal relationships between negative rearing behaviors, social withdrawal, and aggression. The fitness index of the multivariate latent growth models was not sensitive to sample size, and the simplicity indexes such as Tucker-Lewis index (TLI), comparative fit index (CFI), and root mean square error of approximation (RMSEA) were used. For the goodness of fit index, model fitness was considered to be good if TLI and CFI were above 0.90 . Additionally, fitness was considered to be good if RMSEA was below 0.05 , appropriate if below 0.08 , and average if below 0.10 . PASW SPSS ver. 18.0 (SPSS Inc., Chicago, IL, USA) and Lisrel ver. 8.72 (Scientific Software International, Lincolnwood, IL, USA) were used for statistical analyses.

\section{RESULTS}

\section{Changes in Major Variables over Time}

Changes in the levels of negative rearing experiences, social withdraw$\mathrm{al}$, and aggression as the adolescents aged were examined, and the means and standard deviations of the variables at each time point are shown in Table 1. The analysis results showed that in the first and third years negative rearing experiences were maintained at similar levels, and dropped in the fourth year. Social withdrawal showed slight increases in the third year. Aggression showed a slight increase in the

Table 1. Means and standard deviations for major variables

\begin{tabular}{lccc}
\hline \multicolumn{1}{c}{ Variable } & 2nd year & 3rd year & 4th year \\
\hline Negative rearing & $1.857 \pm 0.485$ & $1.842 \pm 0.483$ & $1.803 \pm 0.432$ \\
Social withdrawal & $2.239 \pm 0.706$ & $2.262 \pm 0.738$ & $2.235 \pm 0.692$ \\
Aggression & $2.128 \pm 0.572$ & $2.205 \pm 0.600$ & $1.967 \pm 0.526$ \\
\hline
\end{tabular}

Values are presented as mean \pm standard deviation. third year, and dropped in the fourth year.

\section{Correlations between Major Variables}

To observe relationships between the major variables of negative rearing behavior, social withdrawal, and aggression, correlational analysis was performed between the variables collected at each time point. The analysis results are shown in Table 2 , and there were statistically significant positive relationships $(\mathrm{P}<0.01)$ between all variables measured at each time point.

\section{Analysis Model for Variables}

The model in this study consists of three time points, so a no-change model as in Figure 2 and a linear model as in Figure 3 were selected as competing models.

The no-change model only has the initial value, and the rate of change is not set. This model assumes that there was no significant change over time. The linear model is the most basic form of a latent growth model, and has two latent factors of initial value and rate of change. All factor coefficients of the initial value are set to 1 , and the factor coefficients for the rate of change are set to 0,1 , and 2 . This model assumes linear change over time.

Therefore, the linear model is appropriate for consistent increases or decreases at three time points; and the no-change model is appropriate when the direction of change is inconsistent or when the amount of change is insignificant.

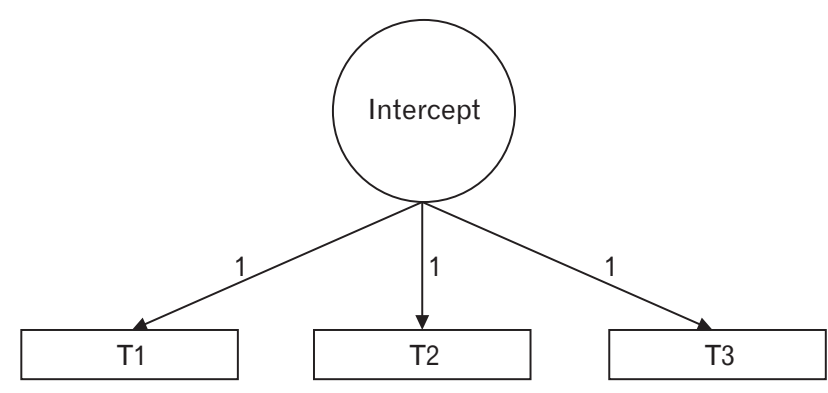

Figure 2. No-change model.

Table 2. Correlations between major variables

\begin{tabular}{|c|c|c|c|c|c|c|c|c|c|}
\hline Variable & 1 & 2 & 3 & 4 & 5 & 6 & 7 & 8 & 9 \\
\hline 1 & 1.000 & & & & & & & & \\
\hline 2 & $0.471^{* *}$ & 1.000 & & & & & & & \\
\hline 3 & $0.393^{\star *}$ & $0.485^{\star \star}$ & 1.000 & & & & & & \\
\hline 4 & $0.118^{* *}$ & $0.067^{\star \star}$ & $0.067^{\star \star}$ & 1.000 & & & & & \\
\hline 5 & $0.133^{* \star}$ & $0.166^{\star \star}$ & $0.101^{\star *}$ & $0.465^{\star \star}$ & 1.000 & & & & \\
\hline 6 & $0.087^{* *}$ & $0.107^{\star \star}$ & $0.133^{\star *}$ & $0.462^{\star *}$ & $0.503^{* *}$ & 1.000 & & & \\
\hline 7 & $0.258^{\star *}$ & $0.167^{\star \star}$ & $0.160^{\star \star}$ & $0.294^{\star *}$ & $0.248^{\star \star}$ & $0.199^{\star \star}$ & 1.000 & & \\
\hline 8 & $0.197^{* *}$ & $0.337^{\star *}$ & $0.201^{* *}$ & $0.239^{* *}$ & $0.335^{\star *}$ & $0.207^{\star *}$ & $0.427^{\star *}$ & 1.000 & \\
\hline 9 & $0.175^{\star *}$ & $0.223^{\star \star}$ & $0.276^{\star \star}$ & $0.247^{\star \star}$ & $0.198^{\star \star}$ & $0.295^{\star \star}$ & $0.380^{\star *}$ & $0.474^{\star \star}$ & 1.000 \\
\hline
\end{tabular}

1 , negative rearing 2nd year; 2 , negative rearing 3rd year; 3 , negative rearing 4th year; 4, social withdrawal 2nd year; 5 , social withdrawal 3rd year; 6 , social withdrawal 4th year; 7 , aggression 2nd year; 8 , aggression 3rd year; 9 , aggression 4th year.

${ }^{* *} \mathrm{P}<0.01$. 
Analysis of the results for the no-change model and linear change model, which were aimed at finding a model that best described the trajectory of each variable, showed that both models had good fitness for negative rearing experiences, social withdrawal, and aggression (Table 3). However, the linear change model showed better fitness, so it was selected as the final analysis model.

As shown in Table 4, the initial average of negative rearing experiences was significant at $1.852(\mathrm{P}<0.001)$. The average rate of change was significant at $-0.024(\mathrm{P}<0.001)$. This indicates that negative rearing experiences that an adolescent recognizes decreased with time at the rate of -0.024 . Furthermore, the initial variance in negative rearing experience was $0.121(\mathrm{P}<0.001)$, and the rate of variance was 0.012 $(\mathrm{P}<0.05)$, which shows that both were significant. This indicates that the level of negative rearing experiences varies depending on the individual adolescent, and the variance over time also depends on the individual as well. The covariance between the initial value and the rate of change $(-0.054, \mathrm{P}<0.001)$ showed a significantly negative relationship. This indicates that during the 8 th grade period, a student with higher levels of negative rearing experiences showed a lower rate of increase, and a student with initially lower negative rearing experiences showed a higher rate of increase as they aged.

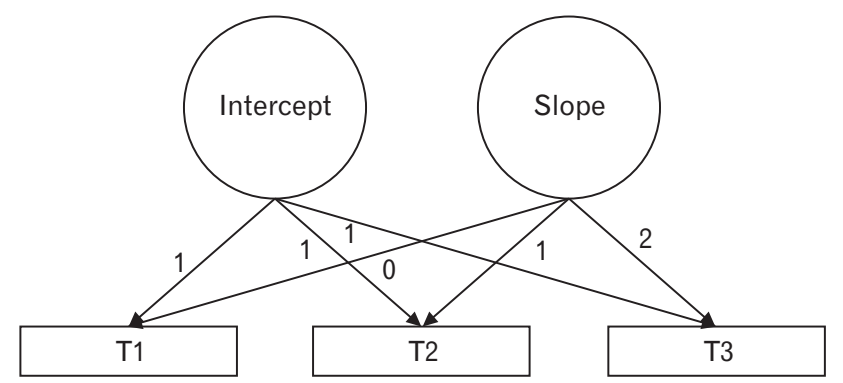

Figure 3. Linear model.
The initial mean for social withdrawal was significant with a value of $2.247(\mathrm{P}<0.001)$, and the average rate of change was -0.003 , which showed a gradual decrease on average; however, this was not statistically significant. On the other hand, the initial variance for social withdrawal was $0.256(\mathrm{P}<0.001)$ and the rate of variance was $0.022(\mathrm{P}<0.05)$, both of which were significant. This indicates that the level of social withdrawal depends on individuals, and the changes over time also depend on the individuals. The covariance between the initial value and the rate of change $(-0.028, \mathrm{P}<0.05)$ had a significantly negative relationship. This indicates that during the 8th grade period, students with higher levels of social withdrawal showed a lower rate of increase, and students with initially lower social withdrawal showed a higher rate of increase as they aged.

The initial average for aggression was significant at $2.181(\mathrm{P}<0.001)$. The average rate of change was also significant at $-0.082(\mathrm{P}<0.001)$. This indicates that adolescent aggression decreases by -0.082 over time. Furthermore, the initial variance of aggression was 0.221 $(\mathrm{P}<0.001)$, and the rate of variance was $0.032(\mathrm{P}<0.05)$, both of which were significant. This indicates that the level of aggression of adolescents may vary depending on the individual, and the amount of change over time also depends on the individual. The covariance between the initial value and the rate of change $(-0.061, \mathrm{P}<0.001)$ had a significantly negative relationship. This indicates that during 8 th grade period, students with higher levels of aggression showed a lower rate of increase, and students with initially lower levels of aggression showed a higher rate of increase as they aged.

\section{Analysis Model between Variables}

The results of the linear change model for each variable were obtained by performing latent model analysis for each of the variables. By setting the research model in Figure 1 as the analysis model, the causal relationship between negative rearing experiences, social withdrawal,

Table 3. Fitness indexes for the no-change model and linear change model

\begin{tabular}{llcccc}
\hline \multicolumn{1}{c}{ Variable } & \multicolumn{1}{c}{ Change model } & $\chi^{2}$ (degree of freedom) & Tucker-Lewis index & Comparative fit index & Root mean square error of approximation \\
\hline Negative rearing experiences & No-change model & $30.908^{\star * \star}(6)$ & 0.990 & 0.980 & 0.045 \\
& Linear change model & $4.465(3)$ & 0.999 & 0.999 & 0.015 \\
Social withdrawal & No-change model & $8.798(6)$ & 0.993 & 0.995 & 0.026 \\
\multirow{3}{*}{ Aggression } & Linear change model & $5.545(3)$ & 0.998 & 0.998 & 0.020 \\
& No-change model & $129.821^{\star * *}(6)$ & 0.943 & 0.891 & 0.089 \\
& Linear change model & $71.779^{\star * *}(3)$ & 0.957 & 0.951 & 0.061
\end{tabular}

${ }^{\star * \star} \mathrm{P}<0.001$.

Table 4. Estimation of developmental trajectories of the main variables

\begin{tabular}{|c|c|c|c|c|c|}
\hline \multirow{2}{*}{ Variable } & \multicolumn{2}{|c|}{ Mean } & \multicolumn{2}{|c|}{ Standard error } & \multirow{2}{*}{ Covariance } \\
\hline & Initial value & Rate of change & Initial value & Rate of change & \\
\hline Negative rearing experiences & $1.852^{\star \star \star}$ & $-0.024^{\star *}$ & $0.121^{\star \star \star}$ & $0.012^{\star \star}$ & $-0.054^{\star \star \star}$ \\
\hline Social withdrawal & $2.247^{\star \star \star}$ & -0.003 & $0.256^{\star \star \star}$ & $0.022^{\star \star}$ & $-0.028^{\star \star}$ \\
\hline Aggression & $2.181^{\star \star *}$ & $-0.082^{\star \star \star}$ & $0.221^{\star \star \star}$ & $0.032^{\star \star \star}$ & $-0.061^{\star \star \star}$ \\
\hline
\end{tabular}

${ }^{* *} \mathrm{P}<0.01,{ }^{* * *} \mathrm{P}<0.001$. 
and aggression was analyzed using a multivariate latent growth model. The fitness calculations showed that TLI and CFI were all above 0.9 as shown in Table 5. RMSEA was below 0.08 , which demonstrates that the model had appropriate fitness.

The research model shown in Figure 1 was set as the analysis model, and the path coefficients between each variable are shown in Table 6 . First, the initial value of negative rearing had a significant effect on the initial values of social withdrawal $(\beta=0.221, t=6.246$; $P<0.001)$ and aggression $(\beta=0.357, t=10.115 ; \mathrm{P}<0.001)$. This indicates that adolescents with higher levels of negative rearing experiences had higher levels of social withdrawal and aggression. Furthermore, the variance of negative rearing had a significant effect on the variances of social withdraw$\mathrm{al}(\beta=370, \mathrm{t}=3.929 ; \mathrm{P}<0.001)$ and aggression $(\beta=0.590, \mathrm{t}=3.700 ; \mathrm{P}<0.001)$. This indicates that as adolescents' negative rearing experiences increased with time, levels of social withdrawal and aggression increased as well. However, the initial value of negative rearing experiences did not have a significant effect on the rates of change in social withdrawal and aggression.

For the effect of social withdrawal on aggression, the initial value of social withdrawal had a significant effect on the initial value of aggression, and the rate of change in social withdrawal had a significant effect on the rate of change in aggression. This indicates that adolescents with higher levels of social withdrawal had higher levels of aggression; if the level of social withdrawal increased with time, aggression increased as well. However, the initial value of social withdrawal did not have a significant effect on the rate of change in aggression.

\section{DISCUSSION}

This study analyzed the effects of negative rearing experiences, due to parental neglect and abuse, on adolescents' social withdrawal and ag-

Table 5. Fitness indexes of the multivariate latent growth model

\begin{tabular}{cccc}
\hline $\begin{array}{c}\chi^{2} \text { (degree of } \\
\text { freedom) }\end{array}$ & $\begin{array}{c}\text { Tucker-Lewis } \\
\text { index }\end{array}$ & $\begin{array}{c}\text { Comparative fit } \\
\text { index }\end{array}$ & $\begin{array}{c}\text { Root mean } \\
\text { square error of } \\
\text { approximation }\end{array}$ \\
\hline $236.100^{\star \star \star}(24)$ & 0.948 & 0.965 & 0.065 \\
\hline
\end{tabular}

$\star * \star P<0.001$. gression. For the analysis, a multivariate latent growth model was used. The study results can be summarized as follows.

Firstly, analysis of the developmental trajectory of negative rearing experiences, social withdrawal, and aggression of adolescents in 8th to 10th grade levels showed that there was a linear trajectory in which the rate of change reduced stably with time. This result can be attributed to the fact that parental influence became relatively reduced due to students spending more time in school work, as they moved to higher grade levels. Furthermore, it can be interpreted to be due to the fact that as students spend more time in school, they have more exposure to peer interactions.

Secondly, the changing patterns in negative rearing experiences, social withdrawal, and aggression depend on individuals, and differences were shown to be clearer in social withdrawal. Such characteristic can show that social withdrawal is a psychological variable that is greatly influenced by personal experiences and the environment. If support from peers or attention from teachers is provided in a timely manner, the probability of adolescents experiencing positive change is likely to be greater compared to the influence of other factors.

Thirdly, the initial value for negative rearing experience had a significant effect on the initial values of social withdrawal and aggression. This indicates that adolescents with negative rearing experience are more likely to experience social withdrawal and aggressive behavior.

Fourthly, the rate of change in negative rearing experience had a significant effect on the rate of change in social withdrawal and aggression. This shows that risk factors hindering adolescent development such as negative rearing experiences, social withdrawal, and aggression do not spontaneously diminish over time, but they persistently act as causal factors impeding adolescent development, continuing to be risk factors into adulthood.

Lastly, the initial value of social withdrawal had a significant effect on the initial value of aggression, and the rate of change in social withdrawal had a significant effect on the rate of change in aggression. This indicates that social withdrawal is related not only to peer and interpersonal interaction, but is also related to low self-esteem and a negative self-concept. Social withdrawal causes anxiety disorders, such as separation anxiety, social anxiety, and phobias, as well as depression, ${ }^{6,27)}$ which can cause antisocial behavior due to maladaptation to

Table 6. Path coefficients of multivariate latent growth models

\begin{tabular}{|c|c|c|c|c|}
\hline Path & Standardized coefficients $(\beta)$ & Standard error & t-value & P-value \\
\hline Initial value of negative rearing $\rightarrow$ initial value of social withdrawal & 0.221 & 0.035 & $6.246^{\star \star \star}$ & 0.000 \\
\hline Initial value of negative rearing $\rightarrow$ rate of change in social withdrawal & 0.018 & 0.020 & 0.873 & 0.383 \\
\hline Rate of change in negative rearing $\rightarrow$ rate of change in social withdrawal & 0.370 & 0.094 & $3.929^{\star \star \star}$ & 0.000 \\
\hline Initial value of negative rearing $\rightarrow$ initial value of aggression & 0.357 & 0.035 & $10.115^{\star \star \star}$ & 0.000 \\
\hline Initial value of negative rearing $\rightarrow$ rate of change in aggression & 0.022 & 0.024 & 0.922 & 0.356 \\
\hline Rate of change in negative rearing $\rightarrow$ rate of change in aggression & 0.590 & 0.160 & $3.700^{\star \star \star}$ & 0.000 \\
\hline Initial value of social withdrawal $\rightarrow$ initial value of aggression & 0.455 & 0.035 & $12.970^{\star \star \star}$ & 0.000 \\
\hline Initial value of social withdrawal $\rightarrow$ rate of change in aggression & -0.019 & 0.022 & -0.865 & 0.387 \\
\hline Rate of change in social withdrawal $\rightarrow$ rate of change in aggression & 0.289 & 0.064 & $4.516^{\star \star \star}$ & 0.000 \\
\hline
\end{tabular}

${ }^{* \star *} \mathrm{P}<0.001$. 
society. ${ }^{28)}$

These study results have the following constructive implications. Firstly, social withdrawal of adolescents derives from neglectful or abusive rearing by parents, creating a risk of behavioral problems of social withdrawal accompanied by anxiety and timidity, due to a loss of a sense of belonging or competence. Such internalizing problems have a high probability of becoming externalized as aggression and delinquency. Most of previous studies on risk factors for impeded adolescent development dealt with the effect of negative rearing experiences on internalizing and externalizing problems such as social withdrawal and aggression; however, there has been insufficient investigation of the relationship with long-term effects. On the other hand, this study examined the developmental trajectory in the relationship between risk factors of adolescent development, from a long-term perspective. Furthermore, the relationship between risk factors was shown to have not only short-term effects but long-term effects as well, which reinforces the limitations of previous studies.

Secondly, parents play a very important role in adolescents' psychological growth. Therefore, abundant positive interactions with parents must be provided. In the long term, there is a need for plans to reduce excessive academic stress on adolescents. Adolescents should receive affection and attention from their parents in order to develop emotional stability. Through correct child-rearing, the self-confidence and social skills of adolescents may be enhanced.

There are several limitations to this study that suggest the following challenges for future research. Firstly, according to previous studies related to withdrawal in childhood, parents play a crucially important role. However, this study has limitations in that only negative rearing experiences were indicated as the factor preceding social withdrawal in adolescents. Social withdrawal is affected by environmental factors including parents, family, and peer interactions. Particularly, adolescents spend relatively increased amounts of time in school, so relationships with peers and teachers can have an influence. Therefore, in future studies, the factors preceding social withdrawal should be comprehensively analyzed.

Secondly, the major variables used in this study derive from the KCYPS data, so additional variables were not sufficiently measured. Therefore, in the future, additional factors that could significantly influence the developmental trajectory of adolescent withdrawal should be explored and examined.

Thirdly, this study analyzed longitudinal relationships between adolescent's negative rearing experiences, social withdrawal, and aggression. However, the relationship between these variables may vary depending on the characteristics of the local community and personal psychological factors. Therefore, future studies should more perform specific analyses on the effects of these factors.

Fourthly, when comparing Western and Asian child-rearing, different normative parenting approaches are generally expected. Such structural differences may have very diverse meanings in the context of the culture. Therefore, there is a need for comparative analysis between Western and Asian styles of child-rearing.

\section{CONFLICT OF INTEREST}

No potential conflict of interest relevant to this article was reported.

\section{REFERENCES}

1. Masten AS, Coatsworth JD. The development of competence in favorable and unfavorable environments. Lessons from research on successful children. Am Psychol 1998;53:205-20.

2. Cicchetti D, Rogosch FA. A developmental psychopathology perspective on adolescence. J Consult Clin Psychol 2002;70:6-20.

3. Pine DS, Cohen P, Brook J. Adolescent fears as predictors of depression. Biol Psychiatry 2001;50:721-4.

4. Rubin KH, Burgess KB, Kennedy AE, Stewart SL. Social withdrawal in childhood. In: Mash EJ, Barkley RA, editors. Child psychopathology. 2nd ed. New York (NY): Guilford Press; 2003. p. 372-406.

5. Bolme-Lake TL. Predicting internalizing problems in at-risk children and adolescents. Boca Raton (FL): Dissertation.com; 2008.

6. Goodwin RD, Fergusson DM, Horwood LJ. Association between anxiety disorders and substance use disorders among young persons: results of a 21-year longitudinal study. J Psychiatr Res 2004;38:295-304.

7. Hoekstra RA, Bartels M, Hudziak JJ, Van Beijsterveldt TC, Boomsma DI. Genetic and environmental influences on the stability of withdrawn behavior in children: a longitudinal, multi-informant twin study. Behav Genet 2008;38:447-61.

8. Oh W, Rubin KH, Bowker JC, Booth-LaForce C, Rose-Krasnor L, Laursen B. Trajectories of social withdrawal from middle childhood to early adolescence. J Abnorm Child Psychol 2008;36:553-66.

9. Attili G, Vermigli P, Roazzi A. Children's social competence, peer status, and the quality of mother-child and father-child relationships. Eur Psychol 2010;15:23-33.

10. Rubin KH, Root AK, Bowker J. Parents, peers, and social withdrawal in childhood: a relationship perspective. New Dir Child Adolesc Dev 2010;2010:79-94.

11. Kiel EJ, Buss KA. Prospective relations among fearful temperament, protective parenting, and social withdrawal: the role of maternal accuracy in a moderated mediation framework. J Abnorm Child Psychol 2011;39:953-66.

12. Ainsworth MD, Blehar MC, Waters E, Wall SN. Patterns of attachment: a psychological study of the strange situation. New York (NY): Psychology Press; 2014.

13. Van Brakel AM, Muris P, Bogels SM, Thomassen C. A multifactorial model for the etiology of anxiety in non-clinical adolescents: main and interactive effects of behavioral inhibition, attachment and parental rearing. J Child Fam Stud 2006;15:568-78.

14. Katz SJ, Conway CC, Hammen CL, Brennan PA, Najman JM. Childhood social withdrawal, interpersonal impairment, and young adult depression: a mediational model. J Abnorm Child Psychol 2011;39:1227-38.

15. Leidy MS, Guerra NG, Toro RI. Positive parenting, family cohesion, and child social competence among immigrant Latino families. J Lat Psychol 2012;1(S):3-13.

16. Perez-Edgar K, Bar-Haim Y, McDermott JM, Chronis-Tuscano A, Pine DS, Fox NA. Attention biases to threat and behavioral inhibition in early childhood shape adolescent social withdrawal. Emotion 2010; 
10:349-57.

17. Raudino A, Woodward LJ, Fergusson DM, Horwood LJ. Childhood conduct problems are associated with increased partnership and parenting difficulties in adulthood. J Abnorm Child Psychol 2012;40:25163.

18. Rinaldi CM, Howe N. Mothers' and fathers' parenting styles and associations with toddlers' externalizing, internalizing, and adaptive behaviors. Early Child Res Q 2012;27:266-73.

19. Yang JW, Kim JH, Oh KJ. Selective attentional bias for negative emotional faces in social anxiety: comparison of three age groups. Korean J Clin Psychol 2006;25:237-55.

20. Chung KM, Shin MJ, Kang JH, Kim ES. Identification of developmental trajectories of social withdrawal in male adolescents: a two-year longitudinal study using growth modeling. Korean J Clin Psychol 2013;32:177-97.

21. Eggum ND, Eisenberg N, Spinrad TL, Valiente C, Edwards A, Kupfer AS, et al. Predictors of withdrawal: possible precursors of avoidant personality disorder. Dev Psychopathol 2009;21:815-38.

22. Lee BJ, Min WH, Kim JE. A study for developmental trajectories of so- cial withdrawal in adolescence: an exploratory approach based on developmental-contextualism perspective. Korean J Youth Stud 2014;21:317-46.

23. Huh MY. The study for the development and validation of 'parenting behavior inventory' perceived by adolescent [dissertation]. Seoul: Ewha Womans University; 2000.

24. Kim SW. The effect of social support on abused children's adjustment [master's thesis]. Seoul: Seoul National University; 2003.

25. Kim SH, Kim KY. Development of behavior problem scale for children and adolescence. J Korean Home Manag Assoc 1998;16:155-66.

26. Cho BH, Lim KH. Development and validation of emotional or behavioral problems scale. Korean J Couns Psychother 2003;15:729-46.

27. Watson J, Nesdale D. Rejection sensitivity, social withdrawal, and loneliness in young adults. J Appl Soc Psychol 2012;42:1984-2005.

28. Serbin LA, Temcheff CE, Cooperman JM, Stack DM, Ledingham J, Schwartzman AE. Predicting family poverty and other disadvantaged conditions for child rearing from childhood aggression and social withdrawal: a 30-year longitudinal study. Int J Behav Dev 2011;35:97106. 